\title{
Comunidad, organización y profesores. Estudio de un liceo rural
}

J oni R. Ocaño*

\section{Resumen}

Este artículo presenta los resultados de un estudio de caso que aborda las relaciones entre organización, profesores y comunidad circundante en un liceo rural que hunde sus raíces en la historia comunitaria. Allí las dinámicas de la organización -instituidas por los profesores más antiguos-, conforman el escenario donde se crean y recrean las prácticas y creencias de los docentes.

Estas dinámicas generan una trama compleja de relaciones y de prácticas que se mantienen y desarrollan alrededor de "los guardianes", modalidad de trabajo que constituye el aspecto central del funcionamiento liceal.

Las distintas ubicaciones de los profesores en ese contexto permiten proponer una clasificación primaria: "progenitores", "adoptantes" y "cohabitantes". Estas posiciones, las estructuras que prosperan con ellas, y sus consecuentes prácticas representan distintas racionalidades que se las ve en conflicto y a la vez como matriz del surgimiento de nuevas prácticas.

\section{Abstract}

This article presents the results of a case study that focuses on the relationships between organization, teachers and surrounding community in a rural secondary school, rooted in the history of the community. The dynamics of the organization -instituted by the oldest teachersare the scenario where teachers' practices and beliefs are created and recreated.

These dynamics generate a complex net of relationships and practices that are kept and developed around the "guardians", a work modality that constitutes the central aspect of the operation of this secondary school.

The different positions of the teachers in that context allow the formulation of a primary classification: "progenitors", "adoptants " and "co-inhabitants". These positions, the structures that prosper with them, and their subsequent practices represent different rationalities that appear in conflict and that are, at the same time, a womb from which new practices emerge.

\section{A modo de exordio}

El investigador esperaba pacientemente en dirección a una profesora para una entrevista no podía atenderlo aún porque estaba en clase-, mientras se entretenía observando las fotos de distintas generaciones de estudiantes y profesores que cubren buena parte de una de las paredes. Ese día el Director estaba en la capital. 
Todo estaba en silencio, la secretaria había salido hacia los salones después de disculparse por no poder acompañar al visitante al tiempo que amablemente le ofrecía: -"quédese cómodo, como en su casa, que enseguida sale la profesora..."

En ese momento el silencio se interrumpió con la llegada de una señora. Parecía apurada, saludó amablemente y se sentó en la silla del director, tomó el teléfono y realizó una llamada mientras miraba al entrevistador con la misma curiosidad que éste la miraba:

-"Hola?,... Ilegaron los de adiestramiento canino-informaba a quien inequívocamente parecía ser el director-, sí... sí, creo que el galpón está disponible..."

El ahora impaciente entrevistador esperó que terminara lo que parecía ser una urgente negociación telefónica, y le preguntó tímidamente:

-"Usted es de aquí..?"

-"Disculpe, estamos apurados, -se justificó antes de contestar- soy vecina de aquí....estamos tratando de resolver un problema, tenemos un experto entrenador de perros y nos faltaba local pero ya resolvimos... ¿y usted... espera a alguien...?"-inquirió con la misma timidez con la que se había iniciado el diálogo-.

Después se supo que se trataba de una ex profesora -ahora jubilada- que se fue a vivir allí, e integra un grupo de fomento de la comunidad. Mantiene lazos fuertemente vigentes con la institución aunque no con el sistema. Es difícil imaginar cuántos convencionalismos y disposiciones reglamentarias se eludieron en ese sencillo trámite, y cuánto se ajustaba a las convenciones particulares de las dinámicas comunitarias e institucionales del caso.

El curso había sido propuesto por los vecinos y el liceo participaba de la organización a través de un equipo - los "guardianes" de animales y mascotas- integrado por algunos estudiantes y un profesor "animador".

Las dinámicas de la institución parecen definirse básicamente con una lógica que integra la concurrencia de dos racionalidades: una relacionada con la comunidad, otra relacionada con el sistema de enseñanza. Ambas, como veremos más adelante, entran en conflicto y parecen generar una nueva racionalidad integradora.

Las instituciones pueden verse como conjuntos precarios de situaciones en equilibrios inestables que hacen posibles y necesarias las dinámicas que las sostienen. Desde cierta perspectiva, una adecuada comprensión de los procesos particulares que se viven en cada institución demanda, entre otras cosas, el estudio del juego de fuerzas que interactúan dentro de las mismas, del entramado de relaciones y de las percepciones de los actores que, conjuntamente con otros factores, le dan forma a la vida institucional y determinan dichos procesos en el terreno particular de los hechos.

La "metodología de los guardianes" es la clave del funcionamiento del liceo al que refiere este estudio. "Los guardianes" es la denominación del proyecto de centro y refiere a los distintos grupos organizados de estudiantes que tienen una responsabilidad específica a su cargo. "Guardianes y no guardias -explica el director-; los guardias cuidan un "objeto", mientras que los guardianes no solo lo cuidan sino que interactúan con él y lo modifican (...) es una idea adaptada de mi experiencia en los Scouts ${ }^{1}$-confesó luego - consiste en grupos organizados de alumnos con una tarea a cargo. Ellos tienen autonomía para resolver y administrar todos los insumos que se requiera y la responsabilidad de dar cuenta de lo realizado...". Se trata de una estructura primaria -instituída por los profesores más antiguos bajo el liderazgo del directorque busca atender a la vez los requerimientos didácticos del aula y las demandas de la comunidad. 


\section{Marco de partida}

a) La educación secundaria rural: incipiencia e inespecificidad.

Ese recorrido muestra que estudios específicos sobre educación rural existen pocos, en general las investigaciones se focalizan en instituciones urbanas. Los pocos casos refieren a educación primaria y la alfabetización de adultos, no a educación de jóvenes o educación secundaria rural en particular.

En todos los casos estos estudios reconocen la especificidad de la educación que se debe brindar en las escuelas rurales, la condición de «ruralidad» es determinante en la definición de la enseñanza para ese medio. Esta especificidad plantea, entre otras cosas, la necesidad de programas diferenciados y de estructuras especializadas para enfrentar los problemas característicos de la educación rural.

Actualmente en el sistema público uruguayo existen tres modalidades de lo que podríamos llamar educación secundaria en el medio rural: una modalidad dirigida más bien a la capacitación técnica y formación para el trabajo rural, ofrecida por instituciones dependientes de la Universidad del Trabajo del Uruguay (U.T.U.); la otra modalidad es la del plan de Educación Postescolar Rural, conocida como «escuelas con plan extendido» que consiste básicamente en la extensión del nivel primario incorporando $7^{\circ}, 8^{\circ}$ y $9^{\circ}$, dependiente del Consejo de Educación Primaria; y la tercera es la de los liceos rurales, experiencia desarrollada a finales de la década de los ochenta y principios de los noventa del siglo pasado, dependiente del Consejo de Educación Secundaria.

b) La organización escolar: diversidad de perspectivas y poca referencia a lo rural.

En cuanto al mesonivel de las organizaciones, en buena parte de la literatura especializada de estos últimos años se ha tomado a la organización escolar como tema central. Los estudios sobre los procesos institucionales, grupales u organizacionales y sus dinámicas internas, sobre todo en la literatura francesa de base estructuralista y psicoanalista, tienen, hoy por hoy, una rica historia. Actualmente se han incorporado otras perspectivas que intentan recoger aportes tanto de la sociología crítica como de la teoría de los sistemas complejos.

Fernández (1998), identifica tres formas habituales de entender el término «institución» según el análisis de la bibliografía en las que aparece: en el sentido de estructuras permanentes, "como sinónimo de regularidad social, aludiendo a normas y leyes que representan valores sociales y pautan el comportamiento de los individuos y los grupos, fijando sus límites". (Fernández, 1998, 13). En el sentido de organizaciones concretas en las que se cumplen ciertas funciones especializadas con el propósito de concretar las acciones y valores propios de la institución (La escuela es un ejemplo). Y en el sentido de "un mundo simbólico en parte consciente y en parte de acción inconsciente", en el que el sujeto se orienta para comprender y decodificar la realidad social.

Habitualmente se considera las organizaciones como "modos concretos en los que se materializan las instituciones" (Butelman, 1996), y se reconoce su carácter contingente -frente a los rasgos de mayor invariabilidad propios de las instituciones- $y$, por lo tanto, el potencial de cambio y de generación de conflictos que representa. 
Con frecuencia los estudios realizados no se han preocupado por distinguir las organizaciones escolares según el contexto inmediato donde se insertan; se han referido más bien a ellas en general tomando como referencia las instituciones urbanas. Si bien es evidente la existencia de elementos invariantes en todas las organizaciones escolares, creemos que el contexto inmediato opera con fuerza en la determinación de las mismas, en su devenir en tanto organizaciones "de libre acoplamiento" al medio, con lo cual la realización de estudios que se focalicen en las instituciones de contexto rural podría ser enriquecedora.

En los últimos años se han desarrollado líneas de investigación que se constituyeron en valiosos intentos de superar algunos reduccionismos propios de las perspectivas más clásicas mediante el análisis de los procesos desde una dialéctica sujeto/estructura. Esto ha permitido el desarrollo de nuevas perspectivas que se caracterizan por considerar la escuela -en tanto organización- como unidad básica del cambio educativo, el contexto probablemente más decisivo para la facilitación o la inhibición de las innovaciones, el ámbito más influyente en la generación de buen número de proyectos de transformación educativa.

Pero la escuela es tomada no sólo como unidad fundamental de la innovación educativa, sino también de procesos y experiencias estables para individuos y grupos. Existe ya una amplia literatura que no duda en afirmar que es la escuela la unidad educativa formal por excelencia, no el aula ni el profesor aislado y particular.

Por otro lado los análisis desde las perspectivas psicoanalíticas han realizado sendas contribuciones al estudio de las organizaciones, fundamentalmente en lo referido al nexo entre realidad psíquica y realidad histórica, al juego de pulsiones antagónicas y la organización social como renuncia a la satisfacción de ellas, además del papel determinante del Gran Hombre $^{2}$ en el edificio social.

De hecho a partir de estas contribuciones se puede decir que no existe sociedad sin mitos de creación, ni organización alguna sin una saga de su creador. Enriquez (1997) concibe la organización como un sistema a la vez cultural, simbólico e imaginario, en el cual se pueden considerar distintos niveles de análisis que abarcan desde lo más general hasta lo más particular. Así se puede partir de una instancia en la cual es esencial el análisis del mito en la construcción de toda organización, hasta la habitualmente omitida por los sociólogos, individual, sin dejar de lado una que atraviesa todas: la instancia pulsional.

\section{c) Los profesores: prácticas y creencias condicionadas.}

En cuanto al micronivel de las interacciones nos interesan las prácticas y creencias de los profesores. Las investigaciones sobre el profesor -sus conductas, pensamientos, creencias, formación, etc.- abundan en todos los sentidos. Existe una tendencia en investigación educativa, a la que se denomina «paradigma de pensamientos del profesor», que se caracteriza por abordar la relación profesores-curriculum desde la subjetividad del docente, o sea, desde las maneras como le dan sentido e interpretan su actividad cotidiana. (Clark y Peterson, 1990; Marcelo, 1987).

Más allá de las distintas perspectivas, resulta evidente que la práctica educativa, en un sentido amplio, no se puede reducir al ámbito de las aulas. A rigor, ni siquiera es posible reducirla a la práctica escolar, ya que existe, paralelamente a ésta, una práctica educativa como experiencia antropológica en sentido cultural general.

\section{4 - Universidad ORT Uruguay}


La práctica educativa -y dentro de ella la escolar-, es esencialmente un producto social, tiene un origen histórico y se enraíza en la experiencia de la colectividad. No se trata del resultado de la acumulación de un saber artesano por parte de los profesores (aunque tiene muchos componentes de tradición): además de nutrirse de destrezas profesionales, se enmarca en una actividad social más amplia; tampoco es un producto tecnológico, o sea, la consecuencia de la aplicación de una determinada teoría científica previa.

Las prácticas profesionales en educación, a menudo no están basadas en investigaciones, conocimientos científicos o teorías. Ello sería incapaz por sí solo de fundamentar todo un modelo educativo dentro de un sistema escolar complejo. Es decir que la realidad de un centro de enseñanza funciona bastante independientemente del conocimiento científico o riguroso. En otras palabras, la práctica educativa es una praxis en la que se entremezclan interacciones sociales muy diversas, cuyos agentes aportan decisivamente sus concepciones, supuestos y valores.

En este trabajo se tomó la expresión "práctica" en el sentido de Feldman quien distingue "práctica pedagógica" - referida a las prácticas en el marco de la clase- de "práctica docente" que "engloba un conjunto diverso de dimensiones relacionadas con el trabajo de los maestros en las escuelas. Estas incluyen múltiples relaciones en el ámbito de la clase, las relaciones institucionales y con la comunidad, la actividad sindical, el involucramiento personal y las representaciones profundas sobre la propia actividad, los procesos de formación, etc." (Feldman, 1993, 29).

\section{El diseño: vicisitudes de un proceso}

Desde el punto de vista metodológico se trata de un estudio de casos que permite llegar a la comprensión de la complejidad y singularidad de esta realidad particular. Se trata de un abordaje fundamentalmente cualitativo que no pretende explicaciones causales, sino comprender un acontecimiento, una realidad única y compleja a la vez, incorporando la perspectiva de los actores.

El estudio se centró en los profesores como uno de los actores clave de las dinámicas de la organización en términos de grupo. Se trata de un pequeño liceo con un plantel docente heterogéneo, del cual se seleccionaron seis para el estudio de sus interacciones y sus acciones particulares en el entramado organizacional teniendo en cuenta las dimensiones comunicativas, políticas y prácticas de dichas acciones.

La observación directa aunada a entrevistas abiertas e "historias de vida" son las técnicas usadas para ello. Esto permitió identificar y comprender la relación entre la organización -con sus espacios, su dinámica particular, su relación con las intenciones de los actores- y las creencias de los profesores sobre la práctica educativa y su ejercitación concreta. Tanto la observación como las "historias de vida" se complementaron con entrevistas.

Se decidió incluir en el diseño metodológico -siempre abierto a ajustes durante el procesouna instancia de aproximaciones exploratorias. Esto responde, por un lado, al enfoque del estudio -más próximo a la idea del desarrollo de un proceso que a la de un diseño terminado a priori-; y por otro, a la relevancia de la información que pretendía recoger para el propio proceso de la investigación. 
La información obtenida -tanto en las instancias referidas a la organización, a la comunidad o a los profesores-, fue sometida a un análisis progresivo desde el propio proceso de investigación. Se trató de ir dando sentido a los datos que se fueron recogiendo en las distintas instancias de la investigación ya que creemos, como Taylor y Bogdan (1987), que "La recolección y el análisis van de la mano" (op. cit, 158).

Sin embargo en el proceso mismo dichas instancias parecen fusionarse ya que sus fronteras se vieron constantemente revisadas y borradas por imposición del devenir natural de los hechos.

Se tocan aspectos de la estructura y de las dinámicas del liceo -con sus rutinas y formas instituidas por los profesores-, ámbitos y estructuras de participación. También se incursiona en la historia de la propia comunidad incrustada recíprocamente en la historia personal de los primeros pobladores, donde se encuentran enraizamientos insospechados de la historia del liceo.

Memoria e identidad: "marcas" de una dinámica propia.

El liceo desde el punto de vista estrictamente edilicio no parece ser ostentoso, ni modificar demasiado el entorno que comparte con otro edificio del mismo estilo arquitectónico: una escuela primaria con la cual está unido físicamente por un patio común.

Una mirada más atenta al edificio revela paredes, espacios, rincones, etc. cargados de trabajos que parecen testimoniar una historia de construcción-reconstrucción y resignificación de dichos espacios. Existen elementos visibles que muestran una preocupación por la memoria de la institución y la identidad comunitaria.

A la entrada del liceo un cartel con el lema "Vale la pena", el cual, a la vez que anuncia al liceo -cuyo nombre adopta de la comunidad-, recibe al visitante con un "Bienvenidos". En el mismo patio de entrada se ven curiosas esculturas de hierro y madera, ambientadas entre bancos de piedra. Sin embargo todo el conjunto está presidido por un vistoso tótem de madera tallado y pintado; en él aparecen en relieve letras y números, símbolos científicos y matemáticos, figuras geométricas, y en dos segmentos del mismo la sigla "LRCP" (aludiendo a su identificación como liceo rural) y el lema "vale la pena" respectivamente. Ese lema se repite también en distintos murales, carteles y hasta en los membretes y documentos de la institución como el proyecto de centro.

Llama la atención una de las pinturas murales tanto por sus dimensiones -ocupa casi toda una larga pared lateral del liceo- como por su simbología y colorido. Ha sido pintada a varias manos, está dividido en cuatro cuadrantes donde se representan distintos aspectos de la comunidad, y cada uno fue pintado por un grupo bien definido: estudiantes, padres, profesores, comunidad.

En otras paredes laterales del edificio se ven otros trabajos de este tipo, de menor tamaño, con imágenes simbólicas y lemas que parecen celebrar la comunidad. Este año los estudiantes de cuarto año están pintando un gran mural en una de las paredes externas en el patio de atrás que imita -según lo explicó uno de sus autores, una entusiasta adolescente- el estilo constructivista de una obra de Torres García. El fondo es todo blanco y sobre él ya están esbozando las primeras figuras -entre ellas el característico mapa de América de sur con el sur hacia arriba- y los primeros colores. Como en la mayoría de los trabajos de generaciones anteriores también aparece el lema "vale la pena", esta vez caprichosamente escondido en un texto que parece jugar con las formas, los espacios y los colores del conjunto. Es necesario mirarlo detenidamente para darse cuenta de tal lema, sus letras -algunas dispuestas intencionalmente al revés- parecen pintadas con el alma juguetona del adolescente.

\section{6 - Universidad ORT Uruguay}


Colgado en la pared de entrada, junto a la puerta principal, a modo de placa recordatoria, hay un viejo disco de arado herrumbrado. El ocre oscuro del gastado disco -producto de la acción de los elementos naturales-, contrasta con una placa de bronce bien bruñido incrustada en su centro donde figura la fecha de inauguración del liceo. "Fue nuestra primera campanilla" -nos explicó más tarde el director- cuando llegó la electricidad compramos un timbre eléctrico, pero los gurises no querían cambiar nuestra campana de arado".

En el interior del edificio se observan nuevos elementos que revelan una preocupación por la historia del liceo, los estudiantes, los profesores y la comunidad. La sala de dirección tiene sus paredes adornadas con fotografías de las generaciones de estudiantes que han pasado por aquí, grupos de profesores y eventos con la comunidad. Año tras año se incorpora una nueva, por lo que el espacio libre de las paredes se va achicando.

En el corredor principal, donde también funciona el comedor, se exhiben trabajos de estudiantes, fotos de grupos de trabajo, de fiestas y eventos que se registran, etc. Tiene dos grandes mesas con caballetes, largos bancos de madera recostados a los lados y algunas mesas más chicas donde se exponen, como en las paredes, diversos trabajos de los estudiantes.

En uno de los dos grandes pizarrones de este corredor se ve dibujado con tiza una gran cuadrícula que contiene los nombres de los integrantes ("guardianes") de los distintos proyectos con sus respectivos coordinadores y animadores. Sobre su derecha en un espacio que ocupa un poco menos de la mitad aparece la leyenda escrita -enmarcada por un sol y el arco iris- en vistosas letras pintadas con tizas de colores: "Llegó la hora de... elegir. Adónde vas?". La artística convocatoria -elaborada en anchas y redondeadas letras, de variado colorido y elaboración prolija-, contrasta con la blanca cuadrícula hecha a mano alzada y cargada de nombres, cuyas numerosas correcciones y enmiendas no logran ocultar la acción del borrador y la sobreescritura dándole a esa zona del pizarrón una tonalidad blanquecina.

Por todas partes hay fotografías, carteles y manualidades elaboradas por los estudiantes que se exponen, hasta los cuadernos de actas y libros de caja de los distintos grupos ("guardianes") están en muestra -sobre una pequeña mesa en el corredor-, al alcance de quien quiera leerlos.

El tan citado lema acompaña la institución desde los años 90, cuando los liceos rurales fueron considerados un proyecto sin futuro por las autoridades de turno diciendo que "no valen la pena" . En esa oportunidad la comunidad liceal vivía el comienzo de una experiencia exitosa, por lo cual decidieron bautizar su proyecto de centro con el lema: "Vale la pena", en claro enfrentamiento con las políticas que se implementaban en ese entonces (como la del plan extendido para algunas escuelas rurales) "Luchamos por lograr la permanencia de nuestros docentes; (...) y los logros han sido conquistas compartidas entre todos. Entre todos, acuñamos el lema de que, a pesar de las penurias, los gurises son nuestra principal recompensa. Por eso reiteramos hasta el cansancio: Señores y Señoras, nuestro liceo vale la pena"4 


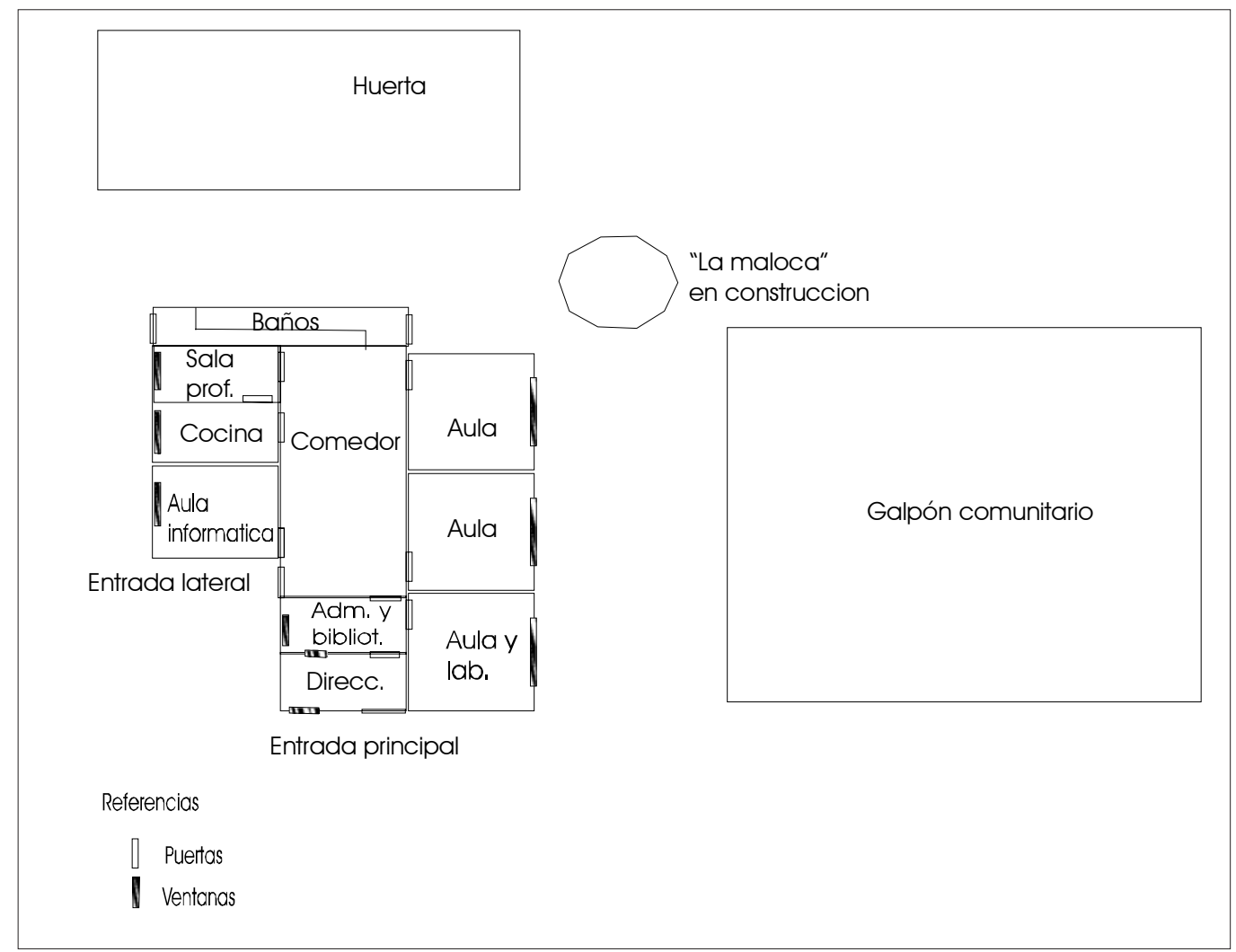

Fig. 1. Planta general del edificio liceal con la disposición aproximada de los ambientes y algunos elementos del entorno.

Todo ese conjunto parece dar cuenta de cierta construcción organizacional, de cierta "mística" liceal, de lucha y conquistas colectivas y celebrar la historia y la identidad de la institución y de la comunidad. "Somos diferentes" es una constante que aparece en los discursos de todos los actores: en el de los profesores y alumnos refiriéndose al modo de trabajar en el liceo, a su organización y especialmente al "método de los guardianes"; en el de los vecinos al compararse con otras comunidades cercanas, en su organización e integración, en la forma como se resuelven los problemas comunitarios y los vínculos entre las distintas instituciones de allí, los diversos proyectos que se promueven y desarrollan a partir de la organización local.

Las cuestiones relacionadas con la comunidad, los proyectos productivos $u$ otros espacios institucionales fuera del aula se resuelven evitando formalismos o disposiciones reglamentarias. Las cuestiones relacionadas con el aula, el aprendizaje, las clases, los programas parece ajustarse a la normativa o a las rutinas propias de cualquier liceo. De esa manera la institución parece definirse básicamente con una lógica que integra la concurrencia de dos racionalidades: una relacionada con la comunidad y las relaciones interpersonales informales, otra relacionada con el sistema. 


\section{La comunidad: historias entrelazadas}

En la comunidad circundante funcionan 15 comisiones vecinales que integran distintas instituciones. Los vecinos parecen estar orgullosos de su comunidad, dicen que las cosas las resuelven ellos y no dependen de ningún "caudillo". Sin embargo reconocen la gravitación de algunos personajes de la zona como uno de los primeros vecinos, -considerado "el alma mater de la comunidad"-, quien tuvo un papel relevante en la creación del liceo, así como en otros logros del centro poblado.

Cuando se trata de eventos comunitarios, todo se hace sin demasiados trámites y, en general, subrayan que aquí las cosas se hacen de forma distinta. Esta relación, donde el liceo es visto como una conquista de la comunidad, hinca sus raíces en el propio proceso de desarrollo comunitario impulsado y promovido por algunos vecinos pioneros que, movidos por ideales (cristianos, cooperativistas, etc.) se vinieron a vivir en la zona y buscaron mejorar las condiciones de vida del lugar.

Las observaciones realizadas parecen indicar que la comunidad tiene un especial "peso" en ciertos aspectos del funcionamiento liceal, fundamentalmente en lo que refiere a los proyectos de integración que promueve el director. Esta percepción es más bien compartida por los profesores que viven en dicha comunidad y los fundadores del liceo, quienes asumen ciertas creencias referidas a la práctica educativa vinculada con los problemas del medio y articulada con su autopercepción de que son distintos. Ellos tienden a considerar la participación de los vecinos a través de los proyectos de producción como dulces, tejido, etc.

Un profesor fundador reconoce en la relación con la comunidad el aspecto más destacable del liceo "... creo que el liceo como motor de la zona, funciona como un centro social donde los vecinos tienen participación". Todo ello es corroborado por la expansión de la participación de los padres en la toma de decisiones en el liceo: desde la A.Pa.L. (Asociación de padres del liceo), órgano institucionalizado por el sistema, y desde el recientemente creado CO.LI.COM. (Consejo Liceal Comunitario), -organismo que decide las inversiones en el liceo- instituido por la organización.

Prácticamente todas las actividades sociales, culturales y algunas productivas se realizan con la participación directa o indirecta del liceo, ya sea con la concurrencia de algún representante del mismo, la utilización de parte de sus instalaciones y recursos (teléfono, etc.), o de la mera difusión de dichas actividades desde el liceo; todo esto como parte de una cultura organizacional propia.

La relación entre la comunidad y el liceo -marcada por una historia particular que los vinculaparece reflejarse hoy en una tensión entre la acción de la comunidad hacia el liceo y la del liceo hacia la comunidad; dicha tensión se resuelve en los "guardianes". Allí convergen las dos racionalidades que sustentan dichas acciones: la de las interacciones personales comunitarias y la del sistema.

El proceso fundacional del liceo está fuertemente unido al desarrollo de la comunidad y, especialmente, a los anhelos de algunos vecinos de la zona. Para éstos la idea original era de un liceo que arraigara a los jóvenes en su comunidad y permitiera a algunos seguir estudiando. En ese sentido se quería una formación en las tradiciones del campo, y una preparación para la producción de la zona. Esos ideales hoy son compartidos por casi la totalidad de los profesores más antiguos en la institución y orientan el sentido del trabajo con los "guardianes". 
Las relaciones contradictorias entre los intereses genuinos del lugar y las políticas educativas que lleva a cabo el sistema aflora en todo momento. Esta misma contradicción se la vive en la propia institución entre la actividad institucional-comunitaria de los "guardianes" y el desarrollo de los programas oficiales en las aulas. Frente al respaldo incondicional a los "guardianes" por parte de los profesores más antiguos un joven profesor entrevistado manifiesta:

-"Conceptualmente es buena la idea de los "guardianes", pero si se exagera termina perjudicando el desarrollo de los cursos. Los aprendizajes de las materias pasan a ser irrelevantes frente a una dinámica muy motivadora pero que deja poco para el aula".

El liceo nace en un entramado complejo de vidas, historias personales, lazos afectivos que implican a personas que, en una buena parte de sus vidas, están o estuvieron ligados directamente a la institución. En ese sentido la creación del liceo se inscribe en un proceso de "maduración" comunitaria y de desarrollo de una comunidad que busca resolver sus problemas locales. Esa es la "marca" fundacional que explica gran parte de las características particulares de la institución hoy.

\section{Los "guardianes": entre la comunidad y el aula}

La conformación de los "guardianes" es voluntaria tanto para estudiantes como profesores quienes eligen en qué proyecto trabajar y los coordinadores de tareas.

Los "guardianes" están organizados en pequeños proyectos que se dividen en proyectos de producción, de gestión y curriculares. Los primeros parecen tener mayor organización y alcance ya que trabajan integrados a proyectos de la comunidad. (Como ejemplos de estos proyectos se podría señalar los "guardianes de huertas y jardines" cuyo funcionamiento -además del cuidado de las flores y de la producción de hortalizas para el comedor- está ligado a la producción de hongos con fines de comercialización integrados a vecinos de la comunidad o los "guardianes de animales y mascotas" que, entre otras cosas producen miel para la comercialización; los "guardianes de biblioteca" cuyo funcionamiento está ligado a la administración del liceo y tienen a su cargo todo el funcionamiento y fomento de la biblioteca; y los "guardianes de laboratorio" o "de salones y material didáctico" cuyo funcionamiento permite apoyar el desarrollo curricular en las distintas áreas).

Según los profesores más antiguos, el funcionamiento de los distintos grupos de trabajo está decayendo. Además consideran que algunos de los nuevos profesores encuentran dificultades al trabajar en esta modalidad; "no comprenden el trabajo con los "guardianes" -manifiesta el director- $y$ algunos no lo respetan porque entienden que interfieren con sus clases normales..."

Sin embargo las actividades de los "guardianes" -algunas articuladas con proyectos productivos mediante convenios realizados con entidades de la zona y con O.N.Gs- conforman el aspecto central de las dinámicas de la organización liceal.

A veces se combina el trabajo de los profesores más antiguos en la institución con el de los más jóvenes, como en la producción de hongos, por ejemplo. En ello se integra el trabajo institucional "extra-aula" -a través de algunos profesores más antiguos involucrados más decididamente con los "guardianes"-; el trabajo de la comunidad -a través de algunos vecinos interesados en producirlos-, y el trabajo "intra- aula" -a través de algunos profesores novatos quienes aprovechan la oportunidad dada para el desarrollo de sus programas en aspectos puntuales (informática con el manejo de los programas de imágenes en el computador, comunicación escrita, matemática, etc.)- aportando al proyecto en el momento de difundir y comercializar el producto.

Al parecer, en el interior de la institución, unos encontraron en esta experiencia la posibilidad de un proyecto que genuinamente representara el nexo con la comunidad, a la vez que orientara la propuesta pedagógica hacia una formación para las actividades 
propias del medio, pero en un marco discursivo "a tono" con las concepciones más difundidas hoy por el sistema. Esto parece plasmarse en uno de los relatos: -"...el trato con los estudiantes es distinto, más directo.(...) Apuntamos más a "la inteligencia emocional" como dice el director. (...) Los proyectos del liceo con la comunidad son vitales ya que con ellos formamos a nuestros alumnos y contribuimos al desarrollo de la comunidad. La comunidad (...) es especial por su integración al liceo". Otros -ya "dentro" de ese marco discursivoencuentran en los "guardianes" una estructura pedagógica "armada" para el desarrollo de sus proyectos curriculares de aula -en una modalidad activa y distinta- coherente con dicho marco. Una joven profesora manifiesta en una entrevista: -"Los guardianes permiten que tú enriquezcas tu aula aprovechando para que los gurises vean los conocimientos en la práctica".

\section{Los profesores: posiciones en la organización}

Teniendo en cuenta las diversas ubicaciones en la organización con respecto a los "guardianes" y su vinculación con ellos, se pudo identificar entre los profesores -por simplificación necesaria de la realidad- dos posiciones fundamentales contrapuestas y una intermedia en transición: la de los educadores que crearon el modelo de los "guardianes" en el liceo, los que lo engendraron instituyendo y conservando con sus prácticas, la forma de trabajo liceal basada en ellos. A estos profesores los llamaremos "progenitores". La de los profesores novatos, que ingresaron recientemente a la institución, que no conocen con profundidad en qué consiste el trabajo con los "guardianes" y desarrollan sus prácticas buscando convivir armónicamente con aquéllas pero sin adoptarlas incondicionalmente. A estos profesores los llamaremos "cohabitantes". Y la de los profesores que tienen cierta antigüedad en la institución, conocen a fondo el trabajo con los "guardianes" y la han adoptado para sí sin ser los que la crearon. A estos profesores los llamaremos "adoptantes".

\section{a) Los "progenitores"}

En general se trata de profesores de mayor antigüedad en la institución, estuvieron desde el origen mismo de la misma. Son fundadores de la modalidad de trabajo que caracteriza y da identidad a la institución. Sus relatos enfatizan en las características de la comunidad circundante, su organización, la buena vecindad, su historia de conquistas (el liceo es una de ellas). Algunos viven en la zona o están vinculados a la comunidad informalmente, mediante sus relaciones de amistad o parentesco, o formalmente, mediante la integración de comisiones en la zona. De esta manera se puede decir que tienen una relación "vital" -o sea mantienen relaciones de su vida personal- con el liceo y la comunidad.

Trabajan impulsados más por una especie de "mandato vital comunitario", las cosas se hacen porque son necesarias para la comunidad o los jóvenes rurales, no porque es obligación profesional. Sus criterios implícitos son más sociales que técnicos. Una profesora contando su experiencia fundadora manifiesta: -"En esa época un grupo de personas luchaban por un ideal real: frenar el éxodo rural y dar la oportunidad de una educación secundaria al joven del campo. Sentí que todos mis ideales tenían una aplicación real y que en lo inmediato podía hacer algo real tangible y no quedar solo en la teoría o en discursos..."

Son defensores de las formas establecidas del trabajo en el liceo y se sienten con autoridad frente a los demás en relación con eso. A su vez se sienten respaldados y valorados por la dirección, los estudiantes y los pobladores del lugar. Actúan, junto a los "adoptantes", como "anfitriones" ante los nuevos profesores, y son los encargados de explicar el funcionamiento de los "guardianes" en la primera asamblea de cada año. 
Sus decisiones las toman, generalmente, respaldados en las formas establecidas históricamente de actuación en la institución -instituida por ellos mismos con sus prácticas cotidianas- y pocas veces buscan respaldos en la reglamentación oficial para la acción.

En su mayoría no tienen formación profesional inicial o la tienen incompleta, sus competencias pasan, al parecer, por el conocimiento del medio, su vocación y entrega personal, más que por la preparación técnica específica.

Todos son coordinadores y/o animadores de "guardianes", están involucrados en los proyectos de la institución, resuelven los problemas más desde la comunicación personal entre pares y desde el sentido común que desde la fundamentación teórica. "Primero hacemos después fundamentamos" manifiesta uno de ellos aludiendo a la necesidad establecida de ello para los registros de la institución. Son informales en sus relaciones con los estudiantes, los vecinos y con los demás profesores. Generalmente están muy bien informados de lo que ocurre en la cotidianidad del liceo y en la comunidad circundante.

Tienen sentimientos ambiguos con respecto a los profesores nuevos: por un lado valoran la juventud y actualización de conocimientos que ellos representan; por otro, ven como amenaza a su trabajo y como problema fundamental el ingreso de muchos profesores nuevos que no conocen la propuesta a fondo. Son más laxos con los horarios, subordinan las aulas al funcionamiento de los "guardianes".

Manejan un discurso técnico homogéneo -buscando integrar lo que traen los nuevos profesores-, al parecer acordado en reuniones como fundamentación de las acciones del liceo (coinciden en las fundamentaciones teóricas) y un discurso privado heterogéneo (en charlas informales se pudo constatar que difieren en sus opiniones personales y de sentido común).

\section{b) Los "cohabitantes"}

Generalmente son jóvenes, titulados en alguna de las modalidades de formación inicial (I.P.A., CE.R.P.) Viven en la capital del departamento y viajan todos los días. Sus relatos enfatizan en las características propias de la organización del liceo, el clima de compañerismo, el espíritu de equipo, la relación con los alumnos, etc. A diferencia de los "progenitores" parecen actuar más bien orientados por una especie de "mandato del sistema", en ese sentido respaldan habitualmente sus acciones en la reglamentación vigente o intentan conocerla para actuar. Con matices, sus vinculaciones con la institución son meramente profesionales, atravesadas por decisiones cuya fundamentación busca ser estrictamente técnica. Las cosas se hacen porque es parte de la tarea, por obligación profesional. Sus criterios implícitos parecen ser más técnicos que sociales.

Algunos buscan mejorar las formas establecidas de trabajo en el liceo tratando de innovar siempre en el marco de los proyectos de los "guardianes". Actúan como aprendices en las cuestiones referidas a los "guardianes" y como "asesores" en cuestiones de planificación de proyectos y fundamentaciones teóricas. Sienten que aportan fundamentación teórica actualizada, son más "duchos" en la elaboración escrita de los proyectos desde un planteamiento netamente formal. En ese sentido establecen relaciones "simbióticas" con los "progenitores" quienes no manejan el nuevo discurso técnico que fundamenta el trabajo con proyectos de centro, pero que les aportan su experiencia y conocimiento práctico para resolver problemas en la institución y en el medio.

\section{2 - Universidad ORT Uruguay}


Sus decisiones las toman, generalmente, respaldados en la normativa o en la autorización de la dirección. Todos tienen formación profesional inicial completa, sus competencias pasan, al parecer, por el conocimiento técnico de la asignatura, del currículo oficial y de las fundamentaciones teóricas de las formas de actuación pedagógica.

Pocos son coordinadores o animadores de "guardianes", están involucrados más en sus proyectos de aula y en los proyectos de la institución que en los comunitarios. Resuelven los problemas más desde el criterio profesional, técnico y fundamentado teóricamente, que desde la comunicación personal entre pares y el sentido común.

En general son más formales en sus relaciones con los colegas, padres y en el aula con los estudiantes. La informalidad que muestran algunos parece ajustarse más a una estrategia profesional de acercamiento que a una actitud vital o propia. Generalmente no están muy informados de lo que ocurre en la cotidianidad del liceo y en la comunidad circundante. Para ello dependen de los más antiguos. En ese sentido perciben una buena participación de los padres en las cuestiones del liceo, pero les preocupa que pocos se interesen por los problemas de estudio de los alumnos en el aula.

Respetan a los profesores "progenitores", valoran la experiencia y el conocimiento práctico de éstos. Son más rígidos con los horarios, priorizan las aulas aunque reconocen valor al funcionamiento de los "guardianes". Cuando les toca, trabajan en los proyectos de "guardianes" pero desde un involucramiento formal, exclusivamente como estrategia didáctica y en relación con contenidos específicos de sus programas.

A diferencia de los "progenitores" son heterogéneos en sus fundamentaciones teóricas de lo que hacen (acuden a discursos que traen de su formación inicial, fundamentados en la didáctica crítica, las teorías cognitivas del aprendizaje, e incluso en teorías lingüísticas y de la comunicación).

\section{c) Los "adoptantes"}

Son los profesores que, por sus características se los puede ubicar entre los dos anteriores. Disponen de una antigüedad relativa en la institución que les permite desempeñarse con comodidad en ella, y, además, tienen las características profesionales de los "cohabitantes" (discurso actualizado, formación técnica, etc.).

Han adoptado, en general, la modalidad de trabajo promovida por la dirección e instituida por los "progenitores".

Sus relatos no hacen énfasis especial en ninguno de los aspectos que enfatizan los "progenitores" o "cohabitantes" -lo comunitario-institucional o lo institucional-áulico-, y más bien destacan a los dos.

En general se trata de profesores que, si bien no fueron fundadores de la modalidad de trabajo que caracteriza y le da identidad a la institución, la conocen y trabajan con efectividad en ella. Sus relatos refieren tanto a las características de la comunidad circundante, su organización, etc.; como a las características propias de la organización del liceo, el clima de compañerismo, la relación con los alumnos, etc. Sin embargo parecen valorar mucho más las cuestiones institucionales. 
Algunos tienen vínculos formales con la comunidad a través de las actividades que el liceo desarrolla. Son los que dominan en número el equipo de gestión, y el apoyo con que cuentan de la dirección se basa más en sus características profesionales que en los lazos de vida compartida previamente como en el caso de los "progenitores".

Trabajan impulsados más por una especie de "mandato institucional", las cosas se hacen porque son necesarias para el buen funcionamiento del proyecto y del liceo, $\mathrm{y}$ consecuentemente porque redunda en beneficio de los estudiantes y la comunidad. Todo lo que beneficia a los estudiantes y mejora el trabajo de los docentes es valorado. Sus criterios implícitos parecen ser más morales que técnicos o sociales.

No defienden incondicionalmente las formas establecidas del trabajo en el liceo pero se sienten cómodos con ellas e intentan mejorarlas. Como los "progenitores", se sienten respaldados y valorados por la dirección, los estudiantes y los pobladores del lugar, pero a su vez son conscientes del marco institucional que los ampara.

Sus decisiones parecen respaldarse en las formas como se resuelven las cuestiones en la institución y en la negociación con los demás, fundamentalmente con el equipo de gestión. También actúan de acuerdo con la reglamentación oficial.

En su mayoría tienen formación profesional inicial, sus competencias pasan, al parecer, tanto por el conocimiento de la institución como por su formación técnica específica.

Son informales en sus relaciones con los estudiantes y con los demás profesores. Están muy bien enterados de lo que ocurre en la cotidianidad del liceo. Algunos se interesan y se informan también de los acontecimientos de la comunidad circundante. Tienen afinidades tanto con los profesores nuevos como con los más antiguos: los primeros por sus vinculaciones dentro del sistema en otras instituciones, los segundos por su experiencia compartida en la institución.

Valoran con igual énfasis las aulas y el funcionamiento de los "guardianes". Manejan un discurso técnico heterogéneo -como los "cohabitantes"-, pero intentan ser conciliadores. Sus discursos privados también son heterogéneos. Algunos son animadores de "guardianes", participando en los proyectos de la institución. Saben conciliar la comunicación personal, el sentido común y la fundamentación teórica.

Todos -"progenitores", "cohabitantes" y "adoptantes"- parecen convencidos de que participan de una experiencia distinta a lo que comúnmente se ve en otros liceos. En el ámbito discursivo aprueban el trabajo de los "guardianes" aunque la valoración y sentido al mismo varía entre ellos de acuerdo con la visión particular que cada uno desarrolla (ver figura 1). 


\begin{tabular}{|c|c|}
\hline & VISIÓN DE LOS "GUARDIANES" \\
\hline PROGENITORES & $\begin{array}{l}\text { Elemento clave. Nexo con la comunidad. Formación } \\
\text { auténticamente rural. }\end{array}$ \\
\hline ADOPTANTES & $\begin{array}{l}\text { Experiencia formativa institucional. "Oportunidad" de desarrollo } \\
\text { del proyecto institucional y curricular. }\end{array}$ \\
\hline COHABITANTES & $\begin{array}{l}\text { Valor pedagógico relativo. Estructura "armada" para } \\
\text { práctica innovadora. Puede interferir en el desarrollo de los } \\
\text { cursos en el aula. }\end{array}$ \\
\hline
\end{tabular}

Sin embargo también representan lógicas distintas dentro de la institución: los "cohabitantes" -respaldados más bien en las disposiciones del sistema, preocupados por los programas oficiales, y el desarrollo de los cursos teóricos en el aula- parecen representar la racionalidad del sistema; los "progenitores" -respaldados fundamentalmente en su experiencia en la institución, en el sentido común y orientados a los aspectos de la comunidad, los "guardianes" $y$ los proyectos productivos- parecen representar la racionalidad del ámbito de la vida comunitaria; los "adoptantes", sin llegar a compartir las experiencias de los "progenitores", están en condiciones, sin embargo, de conciliar cierta experiencia personal en la institución con los beneficios del sistema.

Los "adoptantes" saben aprovechar su experiencia en la institución, parecen haber aprendido sobre el sentido e importancia de esto a la vez que le han aportado elementos propios de su formación académica (fundamentalmente "aggiornando" el discurso teórico de fundamentación de estas prácticas). Parecen representar, con sus acciones y discursos, cierta racionalidad que se da en la intersección entre la lógica del sistema (que define, por tradición, el trabajo docente en relación con el aula) y la lógica del mundo de la vida en la comunidad (que lo concibe en relación con su contexto): la que concibe el trabajo docente en relación con la institución.

Estos grupos se respetan e interactúan con cordialidad aunque se sienten más cómodos en la institución los "adoptantes" en quienes se respalda mucho la dirección y cuentan con la simpatía tanto de los "progenitores" como de los "cohabitantes". Los "progenitores", quienes cuentan con una historia común con el director y con la propia institución, tienen más "sentido de pertenencia" pero no disfrutan de la comodidad de los "adoptantes" en cuanto parecen ubicarse en las antípodas de los "cohabitantes" quienes crecen en número.

La presencia de una gran cantidad de profesores "cohabitantes" genera cada vez más problemas que se intenta sobrellevar con respeto -"... hay muchos profesores nuevos que no tiene ni idea de lo que es este proyecto... eso genera un cierto desequilibrio, este año se agudizó, hubo muchos desentendimientos... Eso sí, nunca hubo falta de respeto entre nosotros..." -manifiesta uno de los "progenitores".

El trato cordial, sin embargo, no oculta el conflicto que representan tales posiciones; más bien funciona como un "acuerdo entre caballeros" tácito, que permite a cada uno construir su identidad, sin contradecir explícitamente al sistema. Se busca resolver los problemas "hacia adentro", sin trascender hacia fuera: las cosas se resuelven "en casa", aunque a veces es difícil ocultarlos aun defendiendo el espíritu de estas dinámicas. 
Por su parte los "adoptantes", quienes se han sumado a esa historia para continuarla y cambiarla aunando "compromiso profesional" y "sentido de pertenencia", parecen coincidir con los primeros en cuanto a la importancia dada a la comunidad -por fuera de una comprensión vital, aunque sí teórica-, pero compartiendo con los "cohabitantes" su visión de lo profesional y lo institucional.

Sin embargo, año tras año son los "cohabitantes" los que crecen en número, acaparando en su conjunto cada vez más horas, aunque individualmente acumulen pocas. Concomitantemente, los "progenitores" -muchos de los cuales no tienen título habilitante- se ven desplazados y su carga horaria disminuida. Además otros se están jubilando.

La consecuencia inmediata de esto es la disminución de la presencia en el liceo de los profesores más antiguos y compenetrados con proyecto de los "guardianes", y el aumento de la presencia de nuevos profesores que no conocen el sentido de dicho proyecto por no haberlo vivido desde su génesis.

El director ve esto con preocupación ya que implica el alejamiento paulatino de los "progenitores", con quienes mantiene lazos personales y son pilares del funcionamiento y la difusión de los "guardianes":

La presencia de los profesores "adoptantes" parece equilibrar las relaciones de poder en la institución donde unos -los "progenitores"- tienen el respaldo de la comunidad y de la institución en la figura del director y otros -los "cohabitantes"- se sienten respaldados por el sistema a través de sus derechos conquistados con la reciente titulación. Los "adoptantes" parecen gozar de ambos beneficios.

\begin{tabular}{|l|l|l|l|}
\hline & $\begin{array}{l}\text { Característica que más } \\
\text { resaltan del liceo }\end{array}$ & $\begin{array}{l}\text { Fuente de } \\
\text { legitimidad }\end{array}$ & $\begin{array}{l}\text { Ámbito principal } \\
\text { de trabajo }\end{array}$ \\
\hline PROGENITORES & Su relación con la comunidad & La comunidad & Guardianes \\
\hline ADOPTANTES & El trabajo institucional & La institución & La institución \\
\hline COHABITANTES & La relación con los alumnos & El sistema & El aula \\
\hline
\end{tabular}

Figura 3- Los profesores según su posición en el liceo.

Algunos no parecen muy comprometidos, entre otras cosas por la baja carga horaria -vienen una vez por semana y tienen un solo grupo- y por no poder participar en las asambleas de los viernes. En esa situación no están solo los "cohabitantes" sino algunos "adoptantes" y uno de los "progenitores" que tuvo que completar horas en otro liceo.

Este liceo es el "espacio" donde se encuentran -y los encuentros no necesariamente son armoniosos- los sueños y los ideales personales y colectivos de una educación para la comunidad de pertenencia, y las determinaciones del sistema que busca ofrecer una educación secundaria rural con el fin de darle continuidad al ciclo escolar habilitando a los jóvenes rurales a continuar estudios. En ese sentido, por un lado parece encuadrarse en el marco de las aspiraciones comunitarias del desarrollo de su identidad, por otro, en las políticas educativas del Estado que buscan atender las inequidades ${ }^{5}$ en relación con el medio rural buscando consolidar la unidad del sistema social. 
De ese encuentro surge, a instancias de la agencia de los sujetos que allí interactúan, una cultura institucional propia, un ethos particular objetivado en las dinámicas de la organización y que constituye lo propio y distintivo del liceo.

El aspecto central de las dinámicas de la organización del liceo son los "guardianes", cuya existencia y funcionamiento parecen concretarse en la intersección entre los ideales comunitarios y los objetivos del sistema. Alrededor de ellos los profesores representan distintas posiciones a partir de su relación y concepción del valor pedagógico de esa estructura genuina de la organización.

Las dicotomías que se presentan en el interior de la organización parecen resolverse en un híbrido donde el rasgo dominante es la ambigüedad: ámbitos formales e informales que, a la vez que parecen excluirse, se complementan y refuerzan permitiendo la existencia y desarrollo de las distintas prácticas y creencias en su interior.

De esa manera "progenitores", "cohabitantes" y "adoptantes" desarrollan sus creencias y prácticas por las cuales se definen, constituyendo una cultura organizacional que les permite cambiar, modificar y modificarse en una suerte de adaptación activa al medio que constituyen y en el cual sobreviven.

La institución es producto de una historia particular nutrida por muchas historias personales; la historia institucional, se enraíza en la comunitaria y está, a su vez, ligada a las vidas de sus actores.

El liceo necesita brindar oportunidades a los jóvenes que decidan quedarse en la comunidad de acuerdo con sus demandas concretas y a los que eventualmente decidan continuar estudios en la ciudad, de acuerdo a la definición del sistema. De esta manera el aula -estructura institucionalizada por el sistema- y los "guardianes" -estructura instituida por los actores- se desarrollan dialécticamente en tal sentido. Las dos racionalidades en conflicto que representan, se actualizan en las dinámicas de la organización y sus vínculos con lo comunitario.

Sin embargo la organización, ese conjunto de relaciones esenciales entre los componentes del sistema (la institución), parece incorporar ambas racionalidades como no excluyentes, ambas parecen necesarias y posibles. Si las condiciones no permiten la posibilidad de una, se las cambia para que el sistema no se extinga.

Los "guardianes" representan ese cambio radical de las reglas de juego para que la institución, en un momento de severos cuestionamientos, pudiera sobrevivir. Las políticas educativas en su momento y la oposición inicial a las formas de acción del liceo de la propia comunidad, constituían el contexto que hacía del liceo rural una experiencia sin futuro (aunque en algunas zonas rurales se iniciaba un crecimiento en la población joven sin posibilidad de continuar estudios), un microsistema necesario pero imposible. Su desaparición era tan inminente como urgente su transformación en su defecto.

El "vale la pena" es, para este caso, a la vez la confirmación y la legitimación de la opción: transformación para sobrevivir. Los actores -impulsados por los más variados interesestransformaron radicalmente las dinámicas de la organización y con ello las propias formas de acción del liceo. 
Sin embargo para sobrevivir hay que hacerlo dentro del sistema que lo crea y da legitimidad. Fuera de él es imposible, con lo cual el cambio radical de las reglas de juego requiere el mantenimiento y desarrollo estratégico del aula y de un discurso legitimador. De esta manera los "guardianes" y las tradicionales aulas comparten un espacio conflictivo -el liceo- donde se desarrolla una dinámica que permite atender tanto a una demanda educativa específica local como a los requerimientos más generales del sistema.

En este caso se evidencia un entorno donde el liceo se desarrolla como producto de las acciones comunitarias históricamente construidas, como "el hijo mimado" de una comunidad una comunidad "progenitora"- que madura a impulso de las necesidades y posibilidades colectivas y, a la vez, de los sueños e ideales personales. Por ser en su génesis una conquista comunitaria, recibe su energía de allí. Su origen está -como la propia maduración comunitariainextricablemente unido a las vidas del lugar. Pero ahora -llegado a su adultez y nutrido de la riqueza que pueden ofrecer esos sueños progenitores y otras perspectivas, sueños e intereses que se incorporan-, también el liceo ofrece energía a su entorno, parecería que intenta incidir en él buscando transformar la matriz que lo generó.

La "simbiosis" es fuerte y el "peso" de la comunidad aunado a esa "mística" de lucha y participación colectiva organizada, implantada por los fundadores del liceo -los "progenitores"liderados por el director -un "hijo adoptivo" de la comunidad, una comunidad también "adoptante"-, parece ser el determinante más importante en el funcionamiento institucional. Dicho funcionamiento, a su vez, conforma la matriz donde se recrean las prácticas y creencias de sus profesores que definen en gran medida ese funcionamiento.

Las dinámicas particulares de la comunidad demandan un funcionamiento de la organización liceal que muchas veces contradice ciertas pautas institucionales definidas por el sistema (piénsese en nuestro exordio). Sin embargo, para la supervivencia misma de dicha organización, se atiende aquellos requerimientos relacionados con el aula cuyo funcionamiento no difiere demasiado de los demás liceos. -"el funcionamiento a nivel aula es el mismo que en todas partes, yo no le noto diferencia,-afirmaba una joven profesora entrevistada- lo que pasa que los guardianes organizan sus horas de reuniones de manera que no interfieran con las horas de clase. Además los guardianes mejoran el funcionamiento del aula porque los gurises están más motivados, y si sabés coordinar los contenidos..."

Alli parecería reeditarse uno de los problemas educativos cruciales de esta época "bisagra": la permanencia, desaparición, o evolución de esa especie llamada educador y de su nicho, la escuela. Las dos esferas que entran en conflicto aquí (el sistema y lo interpersonal) determinan ese problema.

En este caso conviven en precario equilibrio dos especies: una parecería ser la de los profesores-educadores (sean "progenitores", "adoptantes" o "cohabitantes", aunque se cuentan más entre los primeros): aquellos que ejercen una función educadora sin más legitimidad que su talento natural y su vocación y se definen más por esto, aunque puedan gozar de la legitimidad dada por el sistema a través de la titulación; sería una especie en extinción, pero que lucha por mantener su nicho adaptándolo y adaptándose. Otra de las especies parecería ser la de los profesores-funcionarios (sean "progenitores", "adoptantes" o "cohabitantes", aunque se cuentan más entre estos últimos), que si bien pueden participar de aquellas cualidades, se definen más bien por su formación académica en instituciones formales para tales fines. 
La distancia entre profesor y educador es la misma que separa profesiones y vocaciones; son como plantas que crecen y florecen en un conjunto precario de situaciones que las torna posibles y necesarias. Si se destruye ese hábitat la vida no es más posible ni necesaria, se marchita y muere. A no ser que se cambien las reglas del juego y se desarrolle un hábitat donde profesionales con vocación prosperen.

Este liceo en particular se inscribe en una zona donde la producción local está siendo amenazada por la forestación, cuya acción depredadora está diezmando las especies autóctonas y sustituyéndolas por nuevas. Esto está cambiando el hábitat de las primeras. El significado que esto tiene está magistralmente analizado por Rubem Alvez (1985) lo que justifica que dejemos intactas sus palabras aquí:

"Una vez cortada la selva virgen, todo cambia. Es muy cierto que es posible plantar eucaliptos, esa raza sinvergüenza que crece deprisa, para sustituir viejos árboles seculares que nadie vio nacer ni plantó. Para ciertos gustos, queda hasta más lindo: todos alineados, en permanente posición de sentido, preparados para el corte. Y para el lucro. Sobre todo se van los misterios, las sombras no penetradas y desconocidas, los silencios, los lugares aún no visitados. El espacio se racionaliza sobre la exigencia de la organización. Los vientos no serán más cabalgados por espíritus misteriosos, porque todos ellos sólo hablarán de cifras, financiamientos y negocios"'. (Rubem Alvez, 1985: p. 12).

Los vientos del lucro hace mucho tiempo han llegado a la educación; ya no se habla de vocación, solamente se habla de profesión ${ }^{7}$. Los profesores-funcionarios de ahora, habitan un mundo donde nadie tiene rostro, ni nombre, y no hay historias a ser contadas pues no son las personas y sí las funciones lo que cuenta. Por eso pocos los recuerdan a pesar de su proximidad en el tiempo; pasan por nuestras aulas sin dejar rastros. En el mundo que habitan, la educación es algo que se ejecuta en un espacio racional.

Los profesores-educadores, aquellos de nuestra infancia, quienes todos recordamos su nombre y su rostro -porque los tienen así como también poseen una historia a ser contada-, a pesar de estar lejos en el tiempo, están más cerca en la memoria. Estos profesores habitan un mundo donde todos poseen un rostro, un nombre y una historia por contar; donde la educación se construye entre todos en un espacio artesanal.

El profesor-funcionario es alguien que cumple una función en un mundo dominado por el Estado y por las empresas. Es una especie de entidad administrada según su excelencia funcional, la cual siempre se juzga a partir de los intereses del sistema y no de los sueños de las personas. Su tarea es enseñar una asignatura ${ }^{8}$ o disciplina, orientado más a la instrucción o a la información de acuerdo con los requerimientos del sistema, que a la educación ${ }^{9}$, desarrollo o construcción de la persona, de acuerdo con las necesidades humanas.

Por eso el educador es un ausente en el mundo del sistema y más bien se habla de profesor, o de funcionario docente. Tampoco las ciencias hablan mucho de él, han silenciado sobre el educador, se inclinan por hablar del profesor $^{10}$, del docente ${ }^{11}$, o en el mejor de los casos, del profesional de la educación. De esta manera el educador parece tener el estatuto de un concepto utópico, de existencia práctica prohibida y, por esto mismo, existencia teórica imposible. 
Cabría preguntarse sobre la posibilidad del educador en el mundo de hoy. ¿Qué espacio requeriría su desarrollo?, ¿Habría condiciones que hagan posible y necesaria su preparación?. ¿Cuáles serían esas condiciones?, ¿O habría que cambiar las reglas: no se trataría de formarlo y sí de que "despierte?" La necesidad del "despertar" del educador supone un cambio de reglas y un aprendizaje nuevo: tal vez los educadores no se hayan extinguido, pues no han desaparecido, viven aún latentes en la memoria ${ }^{12}$ de un pasado que suele estar muy próximo. Por eso lo que se necesita es que "despierte" (más que "despertarlo"). El profesor-educador pertenece al mundo de la vida, previo a la racionalización de las instituciones, antes de que el sistema lo haya institucionalizado la sociedad ya los había instituido. La institucionalización lo fue borrando (o durmiendo) en favor de la fabricación en serie de los profesores-funcionarios.

Parecería imposible pensar el resurgimiento del educador desde el propio sistema. Pero podría "despertarse" en cada uno de los profesores que se han lanzado a la aventura de construirse colectivamente a sí mismos. A la luz de este estudio parecería que esa aventura solamente sería posible en los intersticios que el sistema deja, a menudo, en las instituciones donde los actores construyen dialécticamente las organizaciones.

En el caso que motiva este trabajo, los "guardianes" parecerían ser la reminiscencia de ese espacio artesanal que se construye entre todos (docentes, alumnos, vecinos). Esto parece ser posible solamente con entrega personal pues de eso se trata, de personas, de singularidades en construcción y autoconstrucción.

Pero también, en ese mismo caso, el aula parecería ser la presencia de ese espacio racionalizado definido por el sistema, hábitat de esos profesores-funcionarios sin rostro -porque para el sistema todos son iguales, como aquellos eucaliptos alineados para el corte- y que, por eso mismo, pueden ser gestionados y sustituidos. En este sentido el liceo resultaría -en su conjunto- un espacio nuevo que integra esa ambigüedad, hábitat donde todos pueden adaptarse activamente adaptándolo. De esa manera "progenitores", "adoptantes" y "cohabitantes" prosperan a su modo y evolucionan en las relaciones "simbióticas" que se instauran, donde todos se benefician de algún modo con el aporte de los demás, en esa cultura compartida de lo exitoso y lo novedoso que todos buscan instituir. Son muchas las señales que conducirían a esa conclusión: los "progenitores" quieren manejar el discurso técnico con la misma fluidez que los "cohabitantes" como forma de legitimar y mejorar sus prácticas; ya piensan en la posibilidad de ir del aula a la comunidad y no solamente de la comunidad al aula (la introducción de nuevos cultivos y hábitos alimenticios en el medio a partir de lo que trabajan en el aula y las charlas a la comunidad es un ejemplo de ello). Otros intentan incorporarse al uso de los soportes tecnológicos con la ayuda de los "cohabitantes". Como dijo una profesora con experiencia en la institución: "Hay un clima de respeto y compañerismo que es muy positivo para todos. En estos dos últimos años se han integrado compañeros nuevos que han dado su aporte con sus conocimientos actualizados. Ellos aportan lo nuevo y nosotros nuestra experiencia, creo que así las cosas pueden funcionar mejor".

Tal vez los "adoptantes" ocupen hoy la posición más adecuada en el momento histórico de la institución; ya son parte de ella y mantienen cierta "distancia" tanto del sistema como de la comunidad con la cual igualmente mantienen lazos sólidos. Esto les permite gozar de cierta autonomía, evitando ser "gestionados" por aquél y "absorbidos" por ésta.

Algunos "cohabitantes", por su parte, intentan coordinar contenidos de sus clases con el trabajo de los "guardianes", lamentando el poco tiempo de que disponen, y quieren aprender aunque también modificar- esa "metodología" particular, como aquél que logró la creación de un nuevo grupo de "guardianes", no vinculado tanto a lo que el medio ofrece sino a la tecnología 
y la informática que se puede ofrecer al medio desde la institución. Algunos vuelven o piensan volver al liceo al año siguiente. Han comenzado a entablar lazos con la comunidad a través de sus alumnos, de las charlas informales con los vecinos propiciadas por la necesidad de amenizar las largas horas de espera por el ómnibus, y de los rutinarios viajes desde y hacia la ciudad compartiendo el mismo transporte. Empiezan a sentirse muy cómodos en un ambiente que consideran "familiar" -a pesar que toman pocas horas en el liceo y que el trabajo resulte exigentey vuelven como si respondieran al llamado de una fuerza interior inexplicable; tal vez estén en busca de su rostro, son los "adoptantes" de mañana y, quien sabe, los "progenitores" de algo nuevo más adelante.

Todos muestran señales de cambio, de aprendizajes; en este caso particular, con esas condiciones, mientras haya espacios para ello, estas personas volverán a poseer un rostro, un nombre y una historia a ser contada. ¿Sería el sustrato que haría necesario y posible la emergencia (¿resurgimiento?) de ese profesor-educador nuevo, capaz de construir con los otros ese espacio de aprendizajes?.

La racionalización le ha quitado la palabra ${ }^{13}$ a los profesores-educadores. Les ha hecho creer que ya está todo dicho, o que no tienen más que decir, callando las voces auténticas de los sujetos quienes, para sobrevivir en ese espacio racionalizado, repiten la voz inauténtica del sistema. El educador debería recuperar su voz y arriesgar todo en la confianza de que la palabra tiene un poder creador; de lo contrario le restaría entonces como única opción el silencio. De esto dependería su sueño y su despertar.

\section{Bibliografía y notas}

Alvez, R. 1985. Conversas com quem gosta de ensinar. San Paulo: Cortez. Autores Associados.

Berger, P.; Luckmann, T. 1995. La construcción social de la realidad. Buenos Aires: Amorrortu. Butelman, I. (comp.) 1996. Pensando las instituciones. Sobre teorías y prácticas en educación. Buenos Aires: Paidós.

Clark, C.; Peterson, P. 1990. Procesos de pensamiento de los docentes. En: WITTROCK, M. La investigación de la enseñanza III. Barcelona: Paidós-MEC. pp. 444 - 531.

Enriquez, E. 1997. A organizaçao em análise. Petrópolis: Vozes.

Feldman, D. 1993. ¿Qué prácticas, qué teorías? Algunas precisiones conceptuales para el estudio de la "práctica docente". En: Revista Argentina de Educación 20(11): pp.26 - 38, 1993.

Fernández, L. M. 1998. El análisis de lo institucional en la escuela. Un aporte a la formación autogestionaria para el uso de los enfoques institucionales. Buenos Aires: Paidós.

Ibañez, J. 1994. El regreso del sujeto. La investigación social de segundo orden. Madrid: Siglo XXI.

Liston, D. P.; Zeichner, K. M. 1993. Formación del profesorado y condiciones sociales de la escolarización. Madrid: Morata.

Magrassi, G. ; ROCCA, M. 1980. La "historia de vida". Buenos Aires: Centro Editor de América Latina S.A.

Marcelo, C. 1987. El pensamiento del profesor. Barcelona: Ediciones CEAC.

Santos Guerra, M. A. 1997. La luz del prisma. Para comprender las organizaciones educativas. Málaga: Ediciones Aljibe. 
Shavelson, R.; Stern, P. 1989. Investigación sobre el pensamiento pedagógico del profesor, sus juicios, decisiones y conducta. En: GIMENO SACRISTÁN, J. ; PÉREZ GÓMEZ, A. La enseñanza: su teoría y su práctica Madrid: Akal/Universitaria. pp. 372 - 419.

Soler Roca, M. 1990. Educación y vida rural en América Latina. Montevideo: IteM.

Stake,R. E. 1999. Investigación con estudio de casos. Madrid: Morata.

Taylor, S. J.; Bogdan, R. 1987. Introducción a los métodos cualitativos de investigación. Buenos Aires: Paidós.

Wittrock, M. 1989. La investigación en la enseñanza. Barcelona: Paidós.

${ }^{1}$ Efectivamente, en la cultura cristiana de los scouts, pasar de una organización de "guardas" o "vigilantes" a una de "guardianes" significó un cambio ideológico sustantivo que tiene que ver con una organización donde la participación y responsabilidades de los distintos grupos son mayores en ésta última.

2 "...el propio Freud siempre trató de encontrar detrás del fantasma, lo que él denominó la roca del acontecimiento. Por eso enuncia en Tótem y Tabú, la hipótesis según la cual, en el origen, existía una «horda primitiva» sometida a un gran macho, que se reservaba el derecho a la posesión sexual de las mujeres. Los hijos desposeídos un día se habrían rebelado y matado al padre. En la secuencia, tomados por remordimientos, ellos habrían idealizado aquel ser y lo habrían convertido en tótem, en ancestro, en un Dios fiador de la comunidad y del amor mutuo..." (Enriquez, 1997, p. 18).

${ }^{3}$ Expresiones de este tipo fueron recogidas oportunamente en algunas entrevistas radiales.

${ }^{4}$ Discurso de inauguración pronunciado por el director en octubre de 2000, y que figura en la Introducción del libro homónimo editado ese mismo año. El subrayado es mío.

${ }^{5}$ Uno de los objetivos de la actual reforma es precisamente la consolidación de la equidad social.

${ }^{6}$ Esta cita está tomada del portugués, la traducción es mía.

${ }^{7}$ Baste ver toda la producción teórica en educación de estos últimos años, gran parte de ella está preocupada por la cuestión de la profesión docente.

${ }^{8}$ Asignatura tiene que ver con signos que a su vez deriva de sigillum: marca o sello. Ibáñez (1994) analiza esto indicando que mediante la enseñanza de una asignatura se marca con sigilo a los alumnos para asignarles una tarea social productiva y no se den cuenta de que están marcados. Enseñar viene de "in" + "signare". Es señalar en su doble sentido: referencial (señalar hacia aquello que se enseña) y gramatical (señalar o grabar en el mismo enseñado una señal, marcarlo como significante, lo mismo que engramar).

${ }^{9}$ Aquí es importante recordar aquella consabida etimología doble del término: en sentido de "ex-ducere" (extraer, hacer salir lo que se tiene en potencialidad), y de "educare" (nutrir, cuidar para que se desarrolle).

${ }^{10}$ Profesar: del latín profitêri, declarar una fe o doctrina. El profesor es el que declara a los demás repitiendo, es la misma raíz de profesión y profesional que alude a esto en términos de actividad remunerativa.

${ }^{11}$ Según Ibáñez (1994) docente viene de doceô: hacer aprender haciendo repetir, comparte la misma raíz de dócil, dúctil que refieren a la capacidad de soportar cualquier torcedura o deformación y "docto"que refiere al que repite lo que le asignaron. 
${ }^{12}$ La pérdida de la memoria es un evento esclavizador: en la novela histórica "Raíces", Kunta Kinte fue vendido a un nuevo dueño que le dio un nombre; esto no es accidental, el primer acto de dominio exige que el dominado olvide su nombre, pierda la memoria de su pasado, no recuerde su dignidad y acepte el nombre que el señor le impone. En fin, desde Platón a Freud el evento liberador exige que seamos capaces de dar nombre a nuestro pasado, algunos autores refieren a la memoria como una experiencia transfiguradora y revolucionaria. Marcuse ha llegado a decir que ella tiene una función subversiva.

${ }^{13}$ La palabra crea. Recordemos que el proceso de socialización comienza en los niños con las palabras. Pero no se trata de cualquier palabra, sino de aquellas que parten de la relación con los otros significantes (Berger y Luckmann, 1995), o sea, palabras auténticas de aquellos que tienen con los niños un destino común. En ese sentido el otro significante debe tener un nombre, un rostro reconocible por los niños y una historia en común con ellos de cuya relación surgen las palabras "eficaces" para dicho proceso.

*Master en Educación, Universidad ORT. Licenciado en Ciencias de la Educación, Universidad Católica del Uruguay. Maestro. Docente, Cursos de Teoría de la Educación e Investigación Educativa Aplicada, Centro Regional de Profesores del Norte. 